\title{
Medical costs of asbestos-related diseases in Spain between 2004 and 2011
}

\author{
M. García-Gómez, R. Urbanos Garrido, R. Castañeda López, A. Menéndez-Navarro \\ (Ind Health. 2017 Feb 7; 55(1): 3-12) \\ DOI: 10.2486/indhealth.2016-0021
}

DOI: 10.12961/aprl.2019.22.01.7

\section{ENTREVISTA CON ALFREDO MENÉNDEZ NAVARROa}

1. ¿Cuál es la procedencia, especialización y áreas de trabajo de los autores del artículo? ¿Qué características le parecen más destacables de este grupo de trabajo?

La característica más destacable del grupo de trabajo es su interdisciplinariedad. Incorpora expertas en salud pública y epidemiología laboral -Montserrat García Gómez y Rosario Castañeda López-, una economista con experiencia en el ámbito de la economía de la salud y los costes de la enfermedad-Rosa Urbanos Garrido-y un historiador de la salud laboral -Alfredo Menéndez Navarro-. Ello nos ha permitido formular preguntas de investigación desde diversas perspectivas e intentar responderlas desde visiones complementarias.

\section{2. ¿Cómo se ha financiado el estudio?}

El trabajo recibió fondos públicos del proyecto "Los riesgos del amianto en España (1960-2002)” (HAR2009-07543, Ministerio de Ciencia e Innovación). Aunque el proyecto finalizó en 2013, el equipo - al que pertenecen dos de los firmantes- ha seguido colaborando para dilucidar preguntas de investigación que no pudieron culminarse durante su ejecución.

\section{3. ¿Qué problema pretende abordar este estudio y dónde radica} su interés o relevancia para la seguridad y salud en el trabajo?

Como es bien conocido, la exposición al amianto en diversos sectores de la actividad productiva ha originado enfermedades que se han puesto de manifiesto, en muchos casos, pasados largos periodos de tiempo. Dado el alto nivel de infra-reconocimiento de esta patología como enfermedad profesional -acreditado y cuantificado en otros trabajos del proyecto-, la mayor parte de las víctimas no han recibido la adecuada compensación y atención específica por parte del sistema de Seguridad Social.

El objetivo de nuestro estudio fue estimar la carga de enfermedad derivada de la exposición laboral al amianto en España, cuantificando el número de enfermedades atendidas en el Sistema Nacional de Salud, y calcular los costes sanitarios directos derivados de su tratamiento para el periodo 2004-2011.

La estimación de la carga de enfermedad y su coste es una herramienta fundamental para adoptar las mejores decisiones de

a. Departamento de Historia de la Ciencia, Universidad de Granada. salud pública, tanto las relativas a la reducción y control de riesgos como las de planificación de los recursos sanitarios, humanos y materiales necesarios.

Su interés o relevancia para la seguridad y salud en el trabajo estriba en que estas enfermedades son evitables promoviendo políticas de prevención de riesgos laborales en los lugares de trabajo. Además, el análisis efectuado nos permite aproximarnos al coste que debería ser asumido por la Seguridad Social (en su cobertura de las enfermedades profesionales) y es trasladado, en cambio, al Sistema Nacional de Salud. La visibilización de los costes contribuye a garantizar una cobertura social adecuada para reparar sus consecuencias.

4. ¿Qué aporta este estudio de novedoso o destacable en relación al resto de producción científica sobre el problema estudiado?

Aunque no contempla todos los costes para la sociedad de estas enfermedades, el estudio pone las bases para un análisis muy poco frecuente en España y que es absolutamente necesario. Una necesidad mayor si cabe dado el grave problema de infra-reconocimiento de los cánceres de origen laboral que existe en España.

Existen aspectos éticos, legales y sociales cuando se evalúan los efectos y la prevención de las exposiciones laborales. Las consecuencias económicas de la morbilidad de origen laboral solo se han investigado en años recientes. Varios estudios indican sin ningún género de duda el enorme coste directo de estas enfermedades en los servicios de salud, así como los costes indirectos por el absentismo, las perdidas en la producción, etc., que ocasionan.

Las estimaciones que presentamos en nuestro estudio son las primeras que se hacen en España sobre este tema y subrayan la importancia del problema del cáncer y otras enfermedades por amianto para los servicios sanitarios.

5. ¿Se han encontrado con alguna dificultad o contratiempo para el desarrollo del estudio?

Las dificultades principales están relacionadas con la ausencia de información primaria a nivel nacional, basada en una auténtica contabilidad analítica, que nos permita hacer un cálculo más preciso de los costes totales derivados del tratamiento de estas enfermedades.

6. ¿Se han obtenido los resultados esperados o se ha producido alguna sorpresa? 
En un estudio de estas características es difícil predecir el resultado (que consiste en cuantificar la magnitud de un coste), pero podríamos decir que no se han producido sorpresas importantes.

7. ¿Cómo ha sido el proceso de publicación? ¿Han sufrido alguna incidencia?

El proceso ha sido el habitual. El artículo fue sometido a la revisión por pares y recibió algunas sugerencias interesantes que nos permitieron mejorar su diseño y redacción. Quizá la enseñanza más destacada en el proceso de publicación de este trabajo es la importancia de identificar bien los intereses de investigación y los ámbitos temáticos de las revistas de la especialidad para que nuestro trabajo tuviera encaje.

8. ¿Qué implicaciones tiene este estudio para la prevención de riesgos laborales? ¿Cuál sería la recomendación para mejorar la práctica profesional en relación al problema estudiado?

El estudio subraya, mediante la cuantificación de sus costes asociados, la importancia de prevenir los riesgos laborales. Este tipo de trabajos debería servir, además, para proporcionar criterios económicos que permitan identificar y priorizar actuaciones o para comparar costes y beneficios de los programas de prevención. Por último, creemos que pueden contribuir a mejorar la identificación, comunicación y reconocimiento de las enfermedades profesionales en España, sin duda una de las asignaturas pendientes de nuestro sistema de salud laboral.

\section{COSTES SANITARIOS DE LAS ENFERMEDADES DERIVADAS DE LA EXPOSICIÓN LABORAL AL AMIANTO ATENDIDAS EN EL SISTEMA NACIONAL DE SALUD ENTRE 2004 Y 2011}

\section{Resumen del artículo}

Fundamento: A pesar de la elevada carga de enfermedad causada por la exposición al amianto en Europa, la estimación de los costes directos e indirectos de la asistencia a los afectados ha recibido escasa atención hasta la fecha. El objetivo de este trabajo fue calcular el coste sanitario de las enfermedades derivadas de la exposición laboral al amianto atendidas en el Sistema Nacional de Salud español entre 2004 y 2011.

Métodos: Para el cálculo de los costes sanitarios directos se utilizaron datos primarios relativos a la atención especializada del Sistema Nacional de Salud (ambulatoria e ingresos hospitalarios) y fuentes secundarias para el coste de la atención primaria de salud y la atención farmacéutica.

Resultados: La atención sanitaria de 37.557 enfermedades por amianto en el Sistema Nacional de Salud en 2004-2011 supuso un gasto de 464 millones de euros. La atención especializada supuso el 50,9\% del coste total, la atención primaria el 10,15\% y la atención farmacéutica el 38,9\%. El coste fue 27,8 veces mayor en hombres que en mujeres. El cáncer de pulmón generó el mayor gasto sanitario, 281 millones de euros.

Conclusiones: El gasto que supone la atención sanitaria de las enfermedades por exposición al amianto tiene un impacto económico negativo para el Sistema Nacional de Salud debido al importantísimo infra-reconocimiento de estas enfermedades por el sistema de contingencias profesionales de la Seguridad Social.
VI CONFERENCIA EUROPEA SOBRE NORMALIZACIÓN Y CERTIFICACIÓN EN EL CAMPO DE LA SEGURIDAD Y SALUD EN EL TRABAJO (EUROSHNET 2019)

12-14 de junio de 2019, Dresde (Alemania)

Información:

Sonja Miesner, KAN Secretariat.

Tel.: +4922412313455

E-mail: miesner@kan.de

Web: www.euroshnet.eu/conference-2019/ 